\title{
Feeding patterns of infants admitted with lower respiratory tract infections in an HIV-endemic area
}

\author{
S Muhomud, ${ }^{1}$ MBBS; K L Naidoo, ${ }^{1,2}$ MB ChB, FC Paed (SA), PhD; V Naidoo, ${ }^{1,2}$ MB BCh, FC Paed (SA), Dip Allerg (SA), Cert
} Pulmonol (SA) Paeds; R Masekela, ${ }^{1} \mathrm{MB}$ BCh, MMed (Paeds), Dip Allerg (SA), Cert Pulmonol (SA) Paeds, FCCP, PhD

${ }^{1}$ Department of Paediatric and Child Health, Nelson R Mandela School of Medicine, Faculty of Health Sciences, University of KwaZulu-Natal, Durban, South Africa

${ }^{2}$ Department of Paediatrics, King Edward VIII Hospital, Durban, South Africa

Corresponding author: S Muhomud (shanah.muhomud@gmail.com)

\begin{abstract}
Background. Lower respiratory tract infections (LRTIs) remain a major cause of mortality and morbidity in infants in South Africa (SA). Exclusive breastfeeding has been shown to decrease mortality and morbidity in infants with LRTIs, especially in the context of HIV/AIDS. Major effort has been put into educating HIV-positive mothers on the benefits of exclusive breastfeeding within effective prevention of mother-to-child transmission (PMTCT) programmes.

Objectives. To determine the feeding practices among infants admitted with LRTIs in an HIV-endemic area.

Methodology. The study was a retrospective chart review of all infants admitted with a diagnosis of LRTI between 1 January 2015 and 31 December 2015 at King Edward VIII Hospital, Durban, SA. Data on feeding patterns, socioeconomic status, maternal HIV status and clinical outcomes of LRTI admissions were collected.

Results. Of the 308 infants enrolled, $63 \%$ were male. Exclusive breastfeeding rates of those aged $<6$ months with LRTIs were higher than those found in previous studies; however, these feeding patterns were significantly associated with the HIV status of the mother ( $p=0.003)$. Mothers who were HIV-negative were twice as likely to breastfeed as those who were HIV-positive (odds ratio 2.25; 95\% confidence interval 1.32- 3.88). There was no association between the type of feeding and the clinical outcomes for LRTIs.

Conclusion. Maternal HIV status influenced the feeding patterns of infants admitted with LRTIs with lower exclusive breastfeeding rates in the HIV-exposed group. Despite extensive efforts to promote breastfeeding within effective PMTCT programmes, HIV-positive mothers are still not harnessing the benefits that exclusive breastfeeding provides.
\end{abstract}

S Afr J Child Health 2018;12(4):154-158. DOI:10.7196/SAJCH.2018.v12i4.1487

Breastfeeding is widely endorsed as the feeding method of choice for newborns and young infants. It is part of the World Health Organization (WHO)'s vital strategy to improve child survival. ${ }^{[1]}$ In addition to providing nutrition to infants, breastmilk plays a key role in conferring protection against infection, including lower respiratory tract infections (LRTIs). ${ }^{[2]}$ The first 6 months of breastfeeding contribute extensively to the modulation of the infant's immune system. ${ }^{[3]}$

Breastfeeding practices have long been been suboptimal in South Africa (SA), and this trend persisted and worsened with the onset of the HIV/AIDS pandemic. ${ }^{[4,5]}$ While $75-97 \%$ of SA women initiate breastfeeding, only $12 \%$ of infants are exclusively breastfed until the age of 3 months, and only $2 \%$ beyond that period. ${ }^{[6]}$ Mixed feeding appears to be the most common feeding pattern, increasing the risk of HIV transmission to the infant. ${ }^{[7]}$ Many factors influence the feeding patterns adopted by mothers. Baby-friendly health facilities are attempting to support mothers in initiating and maintaining breastfeeding. ${ }^{[8]}$ Advice provided by nurses in a health facility, access to running water in the house and receiving advice and support from family have been identified as factors that help to achieve the target of exclusive breastfeeding for up to 6 months. ${ }^{[9]}$ In addition, maternal HIV status has been shown to influence feeding choices. ${ }^{[10]}$

LRTIs are a major public health issue affecting infants, and severe cases often require hospitalisation. The mortality risk in terms of LRTIs in SA remains high among infants who are HIV-infected and HIV-exposed. ${ }^{[11-13]}$ The role of breastfeeding in improving the health outcomes of infants is well documented, and breastfeeding remains a major preventive strategy against acute LRTIs in the infant population. ${ }^{[14-17]}$ Exclusive breastfeeding for 6 months provides protection against respiratory morbidity from LRTIs. ${ }^{[18]}$

In a prospective study, it was found that hospitalisation due to bronchiolitis was twice as high among formula-fed infants than both exclusively breastfed and mixed-fed infants, indicating the protective effect of breastmilk. ${ }^{[14]}$ The protective effect of breastmilk was corroborated when the duration of exclusive breastfeeding was found to be inversely related to the length of hospital stay and the duration of oxygen use in infants with LRTI. ${ }^{[19]}$

SA, like many lower-middle-income countries, follows the WHO recommendations in terms of breastfeeding, as it plays a key role in achieving optimal growth and development, and minimising the risks of acute and chronic infections. ${ }^{[20]}$ Furthermore, the national infant feeding strategy encourages HIV-positive mothers on antiretroviral treatment to exclusively breastfeed their babies up to the age of 6 months. Various priority programmes at community, primary healthcare and institutional levels have invested time, effort and resources in advocating for this strategy.

Formula milk was freely distributed as a measure to prevent motherto-child transmission of HIV through breastmilk, prior to SA adopting the 2009 WHO recommendations on the feeding choices for HIVpositive mothers. ${ }^{[12,17]}$ The practice of providing free formula stopped in 2011. ${ }^{[21]}$ Since the implementation of the new prevention of motherto-child transmission (PMTCT) guidelines in 2010, the practice of exclusive breastfeeding has been emphasised ${ }^{[6]}$ Identifying factors that influence infant feeding practices therefore has important implications for public health, and can influence the outcomes associated with health programmes aimed at promoting child health. 


\section{Objectives}

To determine the feeding practices of mothers whose infants were admitted for LRTIs in an HIV-endemic hospital, in order, firstly, to determine the influence of maternal HIV status on mothers' feeding practices in infants admitted with LRTIs, and secondly, to determine whether there is any association between clinical outcomes of LRTIs in infants, and the type of feeding practice chosen.

\section{Methodology}

The present study was a retrospective chart review conducted in King Edward VIII Hospital (KEH), Durban, KwaZulu-Natal Province (KZN), SA. KEH is a teaching hospital attached to the Nelson R Mandela School of Medicine of the University of KZN, and provides regional and tertiary care in the province, which has a very high HIV prevalence.

The charts of infants admitted to KEH with a diagnosis of LRTI between 1 January 2015 and 31 December 2015 were reviewed.* The WHO definition of LRTI used refers to an outcome definition incorporating at least one specific LRTI sign, reported by a caregiver or the study personnel (fast or difficult breathing, chest

Table 1. Demographic, socioeconomic, HIV and nutritional characteristics of sampled population

\begin{tabular}{|c|c|}
\hline Variable & $n(\%)$ \\
\hline \multicolumn{2}{|l|}{ Age (months) $(N=309)$} \\
\hline$\leq 3$ & $104(34)$ \\
\hline$>3-6$ & $93(30)$ \\
\hline$>6-12$ & $112(36)$ \\
\hline \multicolumn{2}{|l|}{ Gender $(N=309)$} \\
\hline Male & $194(63)$ \\
\hline Female & $115(37)$ \\
\hline \multicolumn{2}{|l|}{ Birth weight $(\mathrm{kg})(N=299)^{*}$} \\
\hline$<2.5$ & $74(25)$ \\
\hline $2.5-3.9$ & $212(71)$ \\
\hline $4.0-4.8$ & $13(4)$ \\
\hline \multicolumn{2}{|l|}{ Gestational age (weeks) $(N=272)$} \\
\hline$\leq 37$ & $50(18)$ \\
\hline$>37$ & $222(82)$ \\
\hline \multicolumn{2}{|c|}{ Nutritional status of infant $(N=300)$} \\
\hline Normal & $265(88)$ \\
\hline Moderate acute malnutrition & $13(4)$ \\
\hline Severe acute malnutrition & $8(3)$ \\
\hline Normal weight but at risk & $14(5)$ \\
\hline \multicolumn{2}{|l|}{ Maternal HIV status $(N=309)$} \\
\hline Positive & $117(38)$ \\
\hline Negative & $192(62)$ \\
\hline \multicolumn{2}{|l|}{ Maternal level education $(N=292)$} \\
\hline Primary school & $12(4)$ \\
\hline High school & $257(88)$ \\
\hline Tertiary & $23(8)$ \\
\hline \multicolumn{2}{|l|}{ Maternal employment $(N=267)$} \\
\hline Employed & $67(25)$ \\
\hline Unemployed & $200(75)$ \\
\hline \multicolumn{2}{|l|}{ Child support grant $(N=279)$} \\
\hline Yes & $117(42)$ \\
\hline No & $162(58)$ \\
\hline \multicolumn{2}{|l|}{ Housing $(N=296)$} \\
\hline Formal & $235(79)$ \\
\hline Informal & $61(21)$ \\
\hline
\end{tabular}

wall indrawing) and/or abnormal auscultatory findings (crackles/ crepitations or bronchial breath sounds). ${ }^{[16]}$ An LRTI was defined as either acute pneumonia or bronchiolitis, according to the senior paediatrician at the time of admission. ${ }^{[16]}$ Infants with cardiac lesions, chronic systemic diseases, neuromuscular disease and congenital abnormalities were excluded. All infants with chronic lung disease were also excluded from this analysis.

Data collected included the variables age, gender, HIV status (mother and infant), feeding practices (exclusive breastfeeding, mixed feeding or formula feeding, and the duration of each), socioeconomic status and birth weight of the infant. Variables related to the following clinical outcome were collected: duration of hospital stay; need for oxygen therapy; need for ventilator support; and mortality.

The Biomedical Research Ethics Committee of the University of KZN granted ethics approval (ref. no. BE027/16).

\section{Statistical analysis}

Descriptive analysis was reported using frequencies and percentages for categorical data. Means and standard deviations were used to describe parametric data, which included length of breastfeeding, birth weight and length of hospital stay. Medians and interquartile ranges were used for all non-parametric data. The associations between risk factors and breastfeeding or clinical outcomes were made using $\chi^{2}$ tests. The Wilcoxon rank sum test was used for the comparison of numeric data between two groups, and the KruskalWallis test for more than two groups. Multivariable adjusted logistic regression was used to assess the association of breastfeeding with clinical outcomes in the presence of other potential confounders/ risk factors. Odds ratios (ORs) and 95\% confidence intervals (CIs) are reported. Data were processed and analysed using Stata 13.0 (StataCorp, USA). $P<0.05$ was considered statistically significant.

\section{Results}

Of the 443 infants who were admitted with acute LRTI during the study period, 358 charts were retrieved, and 309 included in the final analysis, after noting missing charts and exclusions (Fig. 1). Table 1 indicates the demographic and clinical characteristics of the analysed cases in this study. Of the 309 infants, $63 \%$ were male (Table 1). Of the mothers, $38 \%$ were HIV-positive, $74.9 \%$ were unemployed, $41.9 \%$ were receiving social grants, $21 \%$ lived in informal housing and $88 \%$ had completed high school.

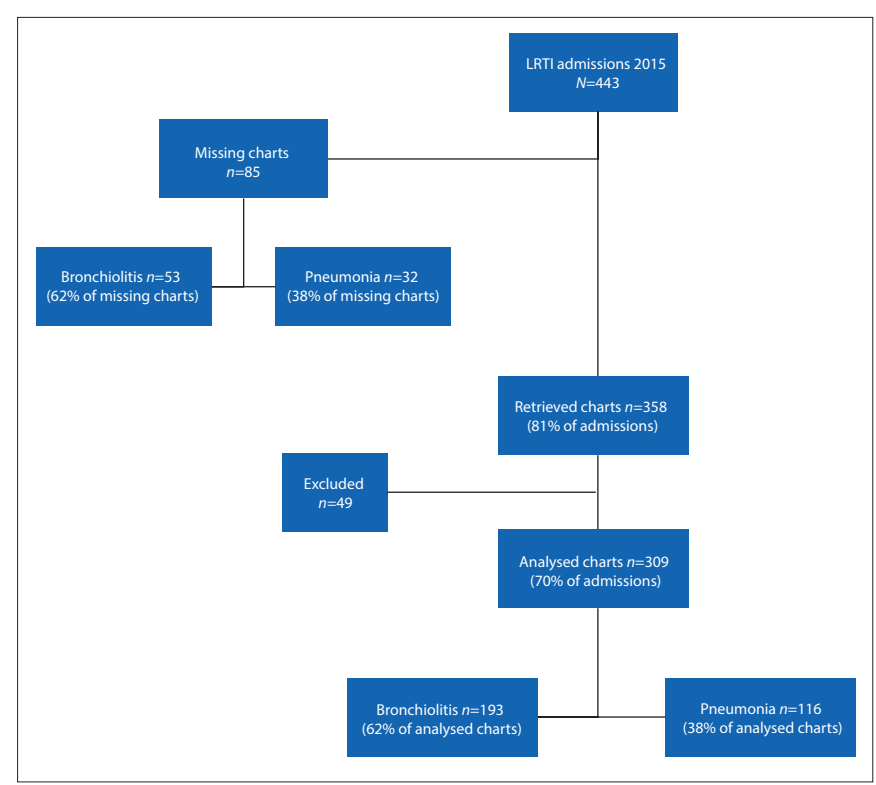

Fig. 1. Infants with lower respiratory tract infections (LRTIs) enrolled in the study. 


\section{Type of LRTIs}

Based on the classification by a senior paediatrician, 62\% $(n=193)$ were diagnosed with bronchiolitis, and 38\% $(n=116)$ with bronchopneumonia (Fig. 1).

\section{Feeding patterns}

Fig. 2 illustrates the feeding choices of analysed cases. Of the 308 infants analysed by feeding pattern, $77 \%(n=238)$ were breastfed at some stage. Of the breastfed infants, 143 (46\%) were exclusively breastfed.

\section{Feeding pattern and clinical diagnosis}

Among the infants who were admitted with bronchiolitis, 89 (46\%) were exclusively breastfed, 56 (29\%) were mixed-fed and 48 (25\%) were formula-fed only. Of those with bronchopneumonia, 54 (47\%) were exclusively breastfed, 39 (34\%) were mixedfed and $22(25 \%)$ were formula-fed. There was no association between feeding pattern and type of clinical LRTI syndrome $(p=0.4)$.

\section{Feeding patterns and}

\section{socioeconomic factors}

There was no association between choice of feeding patterns and grant receipt or maternal education. No inferences could be drawn concerning feeding choice and maternal education, owing to small numbers in the primary and tertiary education groups.

\section{Feeding patterns and maternal HIV status}

When assessing the HIV status of the infants, we categorised infants into two main groups, HIV-exposed and HIV-unexposed. The HIV-exposed group was further subclassified according to the most recent HIV polymerase chain reaction test result available.

Table 2 indicates the different feeding patterns of infants according to HIV exposure.

Fifty-four percent $(104 / 191)$ of all HIVunexposed infants were exclusively breastfed, as compared with only $33 \%$ (39/117) of HIVexposed infants. A larger proportion of HIVexposed infants were mixed-fed or formulafed than HIV-unexposed infants.
When comparing feeding pattern and the HIV status of mothers, the choice of feeding was significantly associated with the HIV status of the mother ( $p=0.003$ ) (Fig. 3). HIVnegative mothers were more than twice as likely to breastfeed as those who were HIVpositive (OR 2.25; 95\% CI 1.32 - 3.88). HIVnegative mothers were also twice as likely to exclusively breastfeed or mix-feed their infants, when compared with HIV-positive mothers (OR 2.4; 95\% CI 1.4 - 4.1).

\section{Clinical outcomes and feeding patterns}

The median lengths of stay were 3 days and 6 days, for infants with bronchiolitis and pneumonia, respectively. When comparing for the median length of hospital stay according to feeding patterns, those of formula-fed, exclusively breastfed and mixed-fed infants were 3,4 and 5 days, respectively, and this was not statistically significant.

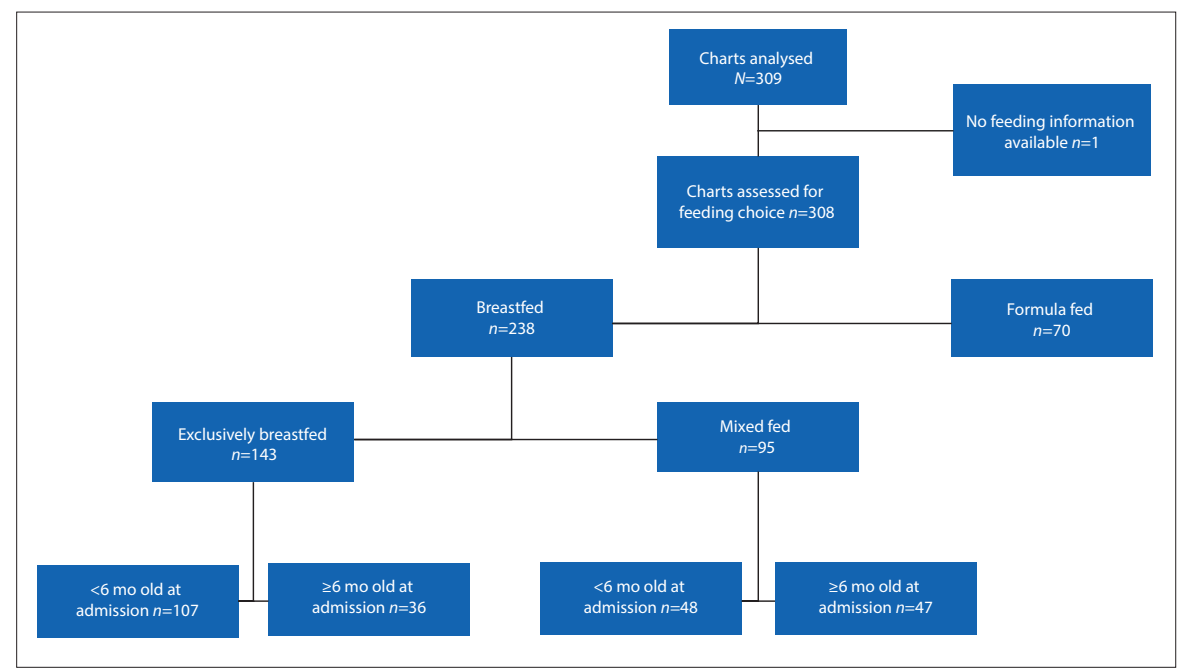

Fig. 2. Classification of all patients according to feeding patterns.

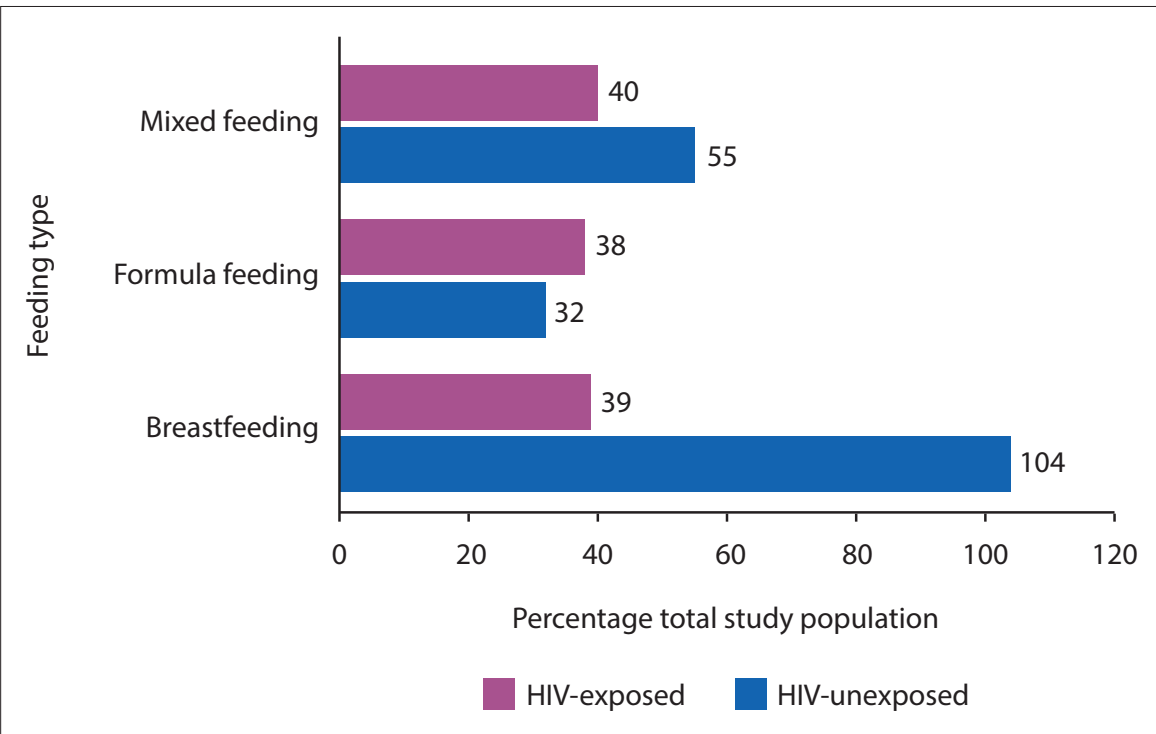

Fig. 3. Feeding patterns among the HIV-exposed and HIV-unexposed infants.

Table 2. Feeding patterns of infants according to HIV status, $\boldsymbol{n}(\%)$

\begin{tabular}{lllll}
\hline HIV status & Exclusively breastfed & Mixed & Formula & Total (\%) \\
\hline Total HIV-unexposed & $104(54)$ & $55(29)$ & $32(17)$ & $191(62)$ \\
Total HIV-exposed & $39(33)$ & $40(34)$ & $38(33)$ & $117(38)$ \\
PCR negative & $24(29)$ & $29(35.5)$ & $29(35.5)$ & $82(27)$ \\
PCR positive & $4(40)$ & $5(50)$ & $1(10)$ & $10(3)$ \\
PCR unknown & $11(44)$ & $6(24)$ & $8(32)$ & $25(8)$ \\
Total & $143(46)$ & $95(31)$ & $70(23)$ & $308(100)$ \\
PCR $=$ polymerase chain reaction. & & & &
\end{tabular}


Patients admitted with acute pneumonia were more likely to require oxygen than those admitted with bronchiolitis $(p<0.005)$. The HIVexposed infants were found to have a longer length of hospital stay (5 days) than HIV-unexposed infants (4 days). The median length of stay among the HIV-exposed infants was longer than that of those who were unexposed after adjusting for feeding pattern (OR 1.59; $95 \%$ CI $1.0-2.4 ; p=0.03)$. There were no associations between the need for oxygen therapy, and HIV status and feeding choice. The numbers were too low to allow for any meaningful comparisons between the exclusively breastfed and formula-fed infants with regard to the need for invasive ventilation.

On regression analysis, HIV-exposure was significantly associated with increased mortality (OR 6.57; 95\% CI 1.03 - 34.2; $p=0.025$ ). HIV-exposed infants were twice as likely to require ventilator support as unexposed infants, but this did not reach statistical significance when adjusted for feeding pattern.

\section{Discussion}

The feeding practices of mothers whose infants were admitted with LRTIs were found to be influenced significantly by maternal HIV status, and this has implications for further programmes related to the care of HIV-exposed infants .

In this cohort, although a high number of infants (77\%) had been breastfed initially, a smaller number had actually been exclusively breastfed. This finding regarding the proportion of exclusive breastfeeding was similar to that in a previous study conducted in SA, which showed that $75-95 \%$ of women initiated breastfeeding at birth, but few continued. ${ }^{[6]}$ A substantial number of mothers discontinued breastfeeding over the subsequent months, suggesting that counselling regarding the benefits of exclusive breastfeeding should be an ongoing process throughout their babies' infancy. It was, however, reassuring that analysis of those infants $>6$ months old indicated that almost one-third (32\%) had been exclusively breastfed up to 6 months, as compared with the findings of the SA National Department of Health in 2003, which showed that only 2\% of SA women exclusively breastfed for 6 months. ${ }^{[6]}$ This may reflect confidence in the PMTCT programme, and improved access to HIV testing, which may provide better information on feeding choices to mothers.

HIV-infected mothers were less likely to exclusively breastfeed their infants, with HIV-infected mothers still having significantly lower rates of breastfeeding than their HIV-negative counterparts. There seems to be a persistent bias towards mixed feeding among HIV-infected mothers, despite the ending of the provision of free formula and the associated education campaigns supporting the use of formula, in 2011. This finding was also noted in a study conducted by Doherty et al. ${ }^{[5]}$ between 2006 and 2008, which showed that in SA, mixed feeding was the most commonly practised type of feeding, even among HIV-infected mothers. This may be related to issues of persistent stigmatisation in the community towards mothers who choose to never breastfeed their infants.

Although this sample reflected overall a population with low socioeconomic indicators, these factors were not as significantly associated with choice of feeding pattern as maternal HIV status.

Many studies have found that breastfed babies have a better outcome than those who are formula-fed, especially with regard to LRTIs. $^{[22]}$ The current study, which focused only on the admitted infants with acute LRTIs, showed no statistically significant associations between the choice of feeding pattern and all clinical measured outcomes. This cohort possibly represents a group with far more serious LRTIs than those in other studies, and therefore these associations may not necessarily be as evident.

HIV-exposed infants remain at high risk of acute LRTIs with longer length of hospital stay and higher morbidity than their unexposed counterparts. This finding is supported by studies that have shown that HIV-exposed infants, though not necessarily infected, were at a greater risk of LRTIs than HIV-unexposed infants. ${ }^{[23]}$ The current study corroborates calls for changes in policy and guidelines to ensure that HIV-exposed uninfected infants be managed as a high-risk group.

Breastfeeding, due to its immunomodulatory benefits, provides protection against many infections, including LRTIs. Studies carried out over the past decade have shown the positive effects of exclusive breastfeeding in terms of lowering infant morbidity and mortality due to LRTI. ${ }^{[22,24,25]}$ In the present study, we could not demonstrate that exclusive breastfeeding was protective against LRTI compared with mixed feeding, but this could be reflective of the cross-sectional nature of the study, which only enrolled inpatients, thereby excluding a large number of children in the community on whom this protection may be conferred.

\section{Study strengths and limitations}

The strengths of this study are that it enrolled a large population of children with LRTIs, in a region with high HIV prevalence and good PMTCT coverage, to assess the impact of feeding practices on LRTIs.

The study is limited by its retrospective nature, and the significant number of missing files. In this cohort, laboratory investigations of LRTIs were not done routinely. Therefore, the LRTIs were categorised largely on clinical criteria. Sputum microscopy and culture, and viral cultures, were not performed. The generalisability of the findings may also be limited to low socioeconomic status communities with high unemployment levels.

\section{Conclusion}

HIV status appears to be a key factor in determining maternal choices in infant feeding practices, as well as their clinical outcomes. Although breastfeeding is initiated in the majority of infants, exclusive breastfeeding rates are reduced by 6 months of life. This could lead to a risk of increased morbidity and mortality among HIV-infected and HIV-exposed, uninfected infants. Programmes to promote exclusive breastfeeding within an effective PMTCT programme need to be reviewed and improved.

${ }^{*}$ The datasets used and analysed in the current study are available from the corresponding author on reasonable request.

Acknowledgements. The authors would like to express their grattitude to Mrs Catherine Connolly for her assistance with the statistical analyses, and Mrs Leora Sewnarain for assistance with typesetting and formatting. Author contributions. SM was responsible for study design, data collection, data analysis and drafting the manuscript. KLN was responsible for supervision of the entire work, study design and manuscript review. RM was responsible for study design, data analysis and manuscript review. $\mathrm{VN}$ was responsible for manuscript review.

Funding. None.

Conflicts of interest. None.

1. World Health Organization, United Nations Children's Fund. Global strategy for infant and young child feeding. Geneva: WHO, UNICEF, 2003. https:// www.who.int/nutrition/publications/infantfeeding/9241562218/en/ (accessed 27 August 2017).

2. Arifeen S, Black RE, Antelman G, Baqui A, Caulfield L, Becker S. Exclusive breastfeeding reduces acute respiratory infection and diarrhoea deaths among infants in Dhaka slums. Pediatr 2001;108(4):e67. https://doi.org/10.1542/ peds.108.4.e67

3. Hanson LÅ. Session 1: Feeding and infant development breastfeeding and immune function. Proc Nutr Soc 2007;66(03):384-396. https://doi.org/10.1017/ s0029665107005654

4. Victora CG, Bahl R, Barros AJD, et al. Breastfeeding in the 21st century: Epidemiology, mechanisms, and lifelong effect. Lancet 2016;387(10017):475490. https://doi.org/10.1016/s0140-6736(15)01024-7 
5. Doherty T, Sanders D, Jackson D, et al. Early cessation of breastfeeding among women in South Africa: An area needing urgent attention to improve child health. BMC Pediatr 2012;12(1):105. https://doi.org/10.1186/1471-2431-12-105

6. Ijumba P, Doherty T, Jackson D, Tomlinson M, Sanders D, Persson L-Å. Social circumstances that drive early introduction of formula milk: An exploratory qualitative study in a peri-urban South African community. Matern Child Nutr 2012;10(1):102-111. https://doi.org/10.1111/mcn.12012

7. Doherty T, Chopra M, Nkonki L, Jackson D, Greiner T. Effect of the HIV epidemic on infant feeding in South Africa: 'When they see me coming with the tins they laugh at me.' Bull World Health Org 2006; 84(2):90-96. http:// www.who.int/bulletin/volumes/84/2/90.pdf?ua=1 (accessed 27 August 2017).

8. Sibeko L, Dhansay MA, Charlton KE, Johns T, Gray-Donald K. Beliefs, attitudes, and practices of breastfeeding mothers from a periurban community in South Africa. J Hum Lact 2005;21(1):31-38. https://doi. org/10.1177/0890334404272388

9. Rollins N, Coovadia HM. Breastfeeding and HIV transmission in the developing world. Curr Opin HIV AIDS 2013; 8(5):467-473. https://doi. org/10.1097/coh.0b013e3283632ba2

10. Thairu LN, Pelto GH, Rollins NC, Bland RM, Ntshangase N. Sociocultura influences on infant feeding decisions among HIV-infected women in rural KwaZulu-Natal, South Africa. Matern Child Nutr 2005;1(1):2-10. https://doi. org/10.1111/j.1740-8709.2004.00001.x

11. Zar HJ, Barnett W, Stadler A, Gardner-Lubbe S, Myer L, Nicol MP. Aetiology of childhood pneumonia in a well vaccinated South African birth cohort: A nested case-control study of the Drakenstein Child Health Study. Lancet Respir Med 2016;4(6):463-472. https://doi.org/10.1016/s2213-2600(16)00096-5

12. Doherty T, Chopra M, Jackson D, Goga A, Colvin M, Persson L-A. Effectiveness of the WHO/UNICEF guidelines on infant feeding for HIVpositive women: Results from a prospective cohort study in South Africa. AIDS 2007;21(13):1791-1797. https://doi.org/10.1097/qad.0b013e32827b1462

13. Slogrove A, Reikie B, Naidoo S, et al. HIV-exposed uninfected infants are at increased risk for severe infections in the first year of life. J Trop Pediatr 2012 May 3; 58(6):505-508. https://doi.org/10.1093/tropej/fms019

14. Lanari M, Prinelli F, Adorni F, et al. Maternal milk protects infants against bronchiolitis during the first year of life. Results from an Italian cohort of newborns. Early Hum Dev 2013;89(S1):S51-S57. https://doi.org/10.1016/ s0378-3782(13)70016-1

15. Cesar JA, Victora CG, Barros FC, Santos IS, Flores JA. Impact of breastfeeding on admission for pneumonia during post neonatal period in Brazil: Nested case-control study. BMJ 1999;318(7194):1316-1320. https://doi.org/10.1136/ bmj.318.7194.1316
16. Roth D. Acute lower respiratory infections in childhood: Opportunities for reducing the global burden through nutritional interventions. Bull World Health Org 2008;86(5):356-364. https://doi.org/10.2471/blt.07.049114

17. Doherty T, Sanders D, Goga A, Jackson D. Implications of the new WHO guidelines on HIV and infant feeding for child survival in South Africa. Bull World Health Org 2010;89(1):62-67. https://doi.org/10.2471/blt.10.079798

18. Oddy W, Sly P, De Klerk N, et al. Breastfeeding and respiratory morbidity in infancy: A birth cohort study. Arch Dis Child 2003; 88(3):224-228. https://doi. org/0.1136/adc.88.3.224

19. Dornelles CT, Piva JP, Marostica PJ. Nutritional status, breastfeeding and evolution of infants with acute viral bronchiolitis. J Health Popul Nutr 2007;25(3):336

20. Shisana O, Rehle T, Simbayi L, et al. South African national HIV prevalence, incidence and behaviour survey, 2012. http://www.hsrc.ac.za/en/research-data/ view/6871 (accessed 27 August 2017).

21. Goga AE, Doherty T, Jackson DJ, et al. Infant feeding practices at routine PMTCT sites, South Africa: Results of a prospective observational study among HIV exposed and unexposed infants - birth to 9 months. Int Breastfeed J 2012;7(1):4. https://doi.org/10.1186/1746-4358-7-4

22. Kaur A, Singh K, Pannu MS, Singh P, Sehgal N, Kaur R. The effect of exclusive breastfeeding on hospital stay and morbidity due to various diseases in infants under 6 months of age: A prospective observational study. Int J Pediatr 2016; 7647054:1-6. https://doi.org/10.1155/2016/7647054

23. Weinberg A, Mussi-Pinhata MM, Yu Q, et al. Excess respiratory viral infections and low antibody responses among HIV-exposed, uninfected infants. AIDS 2017;31(5):669-679. https://doi.org/10.1097/qad.0000000000001393

24. Duijts L, Jaddoe VWV, Hofman A, Moll HA. Prolonged and exclusive breastfeeding reduces the risk of infectious diseases in infancy. Pediatr 2010;126(1):e18-e25. http://doi.org/10.1542/peds.2008-3256

25. Lanari M, Adorni F, Silvestri M, Coscia A, Musicco M. The multicenter Italian birth cohort study on incidence and determinants of lower respiratory tract infection hospitalisation in infants at 33 weeks' GA or more: Preliminary results. Early Hum Dev 2011;87(Suppl):S43-S46. https://doi.org/10.1016/j. earlhumdev.2011.01.009

Accepted 24 April 2018. 